P206 (continued)

Evaluation: Year-end metrics show annual growth in mobile device users for the nutrition and food safety websites, including 19\% increase for nutrition and 52\% increase for food safety. Current websites are not responsive to mobile devices. Analytics reveal parallels across websites' audiences, including $71 \%$ and $66 \%$ female and $29 \%$ and $34 \%$ male for nutrition and food safety, respectively. Similarity among age distribution was also confirmed. Consumer input was not included in original design process on either website.

Conclusions and Implications: Research indicates consumers sought out evidence-based nutrition and food safety resources and stayed connected via social media. More mobile users suggests need for a mobile responsive site. Similar audiences and intervention goals between websites reveal need for user-directed consolidation of nutrition and food safety topics in one updated website that will address food-centered needs of populations. Updates will improve user-friendliness, search engine rankings, and time management for user and programmers.

Funding: None.

\section{P207 The Role of Peer Influence on Food Choice Behaviors in Social Media Platform Environments: A Scoping Review}

Alicia Chung, EdD, MPH, alicia.chung@nyumc.org, NYU School of Medicine, 227 E 30th Street, New York, NY 10016; Azizi Seixas, PhD; Dorice Vieira, MPH;

Nick Tan, BS, City University of New York;

Rafey Iqbal, BS, NYU School of Medicine; Kevin Chery, BS; Gbenga Ogedegbe, MPH, MD

Background (Background, Rationale, Prior Research, and/or Theory): Peer influence on health behaviors is well documented in face-to-face interactions. Little is known about this influence in virtual social media environments.
Objective: To conduct a scoping review to determine the influence of peer influence via virtual social media platforms on food choices in adolescent youth.

Study Design, Setting, Participants, Intervention: Research questions were developed using the PICO framework, and the review was structured using PRISMA guidelines. Preliminary MeSH search terms in PubMed and Emtree terms in Embase along with keywords determined search terms based on adolescent age group, social media platforms, including Twitter, Facebook, YouTube, Pinterest, Instagram, Snapchat and Whatssapp, and eating behaviors. PubMed/Medline, Embase, FSTA, INSPEC, CINAHL, PsycINFO, ERIC, GoogleScholar and Web of Science databases were searched from inception to date. Grey literature and hand searches were conducted as well. Outcome Measures and Analysis: After screening 1,380 peer-reviewed articles, 90 were discordant processes, 17 articles were included as background information, and seven articles met the final inclusion criteria.

Results: Social media volume was measured by user accounts or unique username accounts in observational studies. Intervention studies included a final sample of 431 children and adolescents, 10 to 19 years old, across the United States, England, Sweden, Portugal, and Australia engaged in an online social media platform that influenced their eating behavior. Peer networking influence in a social media environment was found to influence education and behavior change in five thematic areas: eating disorders; food safety; fruit and vegetable intake and weight loss; marketing branded products; and purportedly supportive disease conditions.

Conclusions and Implications: Peer influence on behavior has been well established in family, school and neighborhood social networks. The contagion effect of social media among adolescent youth serves as a medium to market behavior change disseminating messages that influence the influencers towards healthful food choices.

Funding: NIH. 